\title{
Design of Arc Fault Detection Circuit in Low Voltage Switchboard
}

\author{
Kuan Lee Choo and Ahmad Azri Sa'adon \\ Faculty of Engineering and Technology Infrastructure, Infrastructure University \\ Kuala Lumpur, Selangor, Malaysia \\ E-mail:lckuan@iukl.edu.my; azriaces@gmail.com
}

Received 5 October 2016; Accepted 17 November 2016;

Publication 26 November 2016

\begin{abstract}
This paper presents the design of the arc fault detection circuit that is able to detect three different signals prior to the occurrence of an arcing fault in the low voltage switchboard. There are pressure, heat and light signals. The simulation results show that the proposed arc fault detection circuit will activate the relay and send a trip signal to the circuit breaker if the illumination level in the interior of the low voltage (LV) switchboard is more than a predetermined value and at the same time both the pressure and temperature detectors detect a pressure and temperature level which are higher than the reference value. This is to ensure that no fault tripping signal is sent to the circuit breaker and therefore avoid unnecessary power shut down.
\end{abstract}

Keywords: LM 335 Temperature Sensor, 1140 Pressure Sensor, ISL2910 Light to Analog Sensor, Arc Fault, Low Voltage Switchboard.

\section{Introduction}

An arc fault is a high power discharge of electricity between two or more conductors. This discharge translates into heat, which can break down the wire's insulation and possibly trigger an electrical fire. These arc faults can range in power from a few amps up to thousands of amps high and are highly

Journal of Industrial Engineering and Management Science, Vol. 1, 1-16.

doi: 10.13052/jiems2446-1822.2016.005

(c) 2016 River Publishers. All rights reserved. 
variable in terms of strength and duration. Common causes of arc faults include faulty connections due to corrosion and faulty initial installation. The number of incidents related to arcing fault began to rise in the 1960's when the field power system undergoes rapid development to meet the increasing of load demand [1].

Arc incidents occur due to various reasons such as, poorly installed equipment (human mistakes), natural aging of equipment, bad connections, faulty connection due to corrosion. Statistics have shown that $80 \%$ of electrically related accidents and fatalities involving qualified workers are caused by arc flash or arc blast. A true arc fault will rapidly increase energy level up to $20 \mathrm{MW}$ cycle, increase in pressure up to $3 \mathrm{~atm}$, and in heat up to 3000 degrees Celsius [2].

An arcing fault instantaneously release large amount of radiant energy. The radiant energy includes both light and thermal energy. Light intensities can be thousands of time higher than normal ambient light [3]. The light sensor is incorporated in the arc fault detection circuit as light is relatively easy to detect [4]. The main disadvantage of a light detection circuit is the risk of tripping from a light source not related to an arc flash. However, this risk can be reduced to minimum in this proposed design that incorporates three different signals prior to the occurrence of an arcing fault in the low voltage switchboard. Therefore, it is able to ensure no fault tripping signal is sent to the circuit breaker and detect the possibility of arc fault occurrence at the early stage.

This paper is organized as follows: Section 2 provides the study of behavior and characteristic of the arc fault. Section 3 subsequently presents the system description of the arc fault detection circuit. Section 4 describes the modeling of the arc fault detector circuit. Section 5 provides the simulation results of the arc fault detection circuit. Lastly, Section 6 concludes the finding of this paper.

\section{Behaviour and Characteristic of Arc Fault}

An arc fault is the discharge of electricity through the air between two conductors which creates huge quantities of heat and light. It is a high resistance fault with resistance similar to many loads and it is a time varying resistor which can dissipate large amount of heat in the switchboard [5].

Circuit breakers are tested by bolting a heavy metallic short across the output terminals to determine their capabilities of handling an essentially zero resistance load. The zero resistance faults are named as bolted fault. 
Bolted fault current is the highest possible current supplied by the source and a protective system is designed according to the value of bolted fault current. The protective system must be able to detect the bolted fault and the protective devices must be capable of interrupting this value of current [6].

Due to the high resistance loads, an arcing fault will result in much lower values of current. The arcing fault current is often insufficient to operate the protective devices such as circuit breakers, fuses and relays. As a result, the arcing fault will persist until severe burn down damage occurs. The magnitude of the arc current is limited by the resistance of the arc and the impedance of the ground path [7].

Arc faults are categorized into series arc faults and parallel arc faults. Series arc faults happen when the current carrying paths in series with the loads are unintentionally broken whereas parallel arc faults happen between two phases, phase to ground or phase to neutral of the switchboard [8].

Large amounts of heat will be dissipated during an arc event. A portion of this heat is coupled directly into the conductors, a portion heats the air and another portion is radiated in various optical wavelengths. Hasty heating of the air and the expansion of the vaporized metal into gas produces a strong pressure wave which will blow off the covers of the switchboards and collapse the substations [8].

Figure 1 shows the time, current and damage for the 53 arcing tests. When the circuit breakers are tripped within less than 0.25 seconds, the damage will be limited to smoke damage. The triangle markers represent arcs that cause only smoke damage to the side of switchboards. The square markers represent arcs that cause surface damage to the side of switchboards whereas the star pointers represent holes of several square inches at the side of the switchboards [5].

When an arc is ignited, the plasma cloud expands cylindrically around the arc. The expansion of the plasma is constrained by the parallel busbar and thus the plasma expands more to the front and the back of the bus. As the plasma reaches any obstructions such as the switchboard, plasma expansion is retarded by the obstructions. Due to the lower velocity of the arc, the plasma becomes more concentrated and its temperature and current will increase [5].

The root of the arc where the arc contacts the conductor is reported to reach temperatures exceeding $20000^{\circ} \mathrm{C}$, whereas the plasma portion or positive column of the arc is around $13000^{\circ} \mathrm{C}$ [9]. For reference, surface of the sun is reported to be about $5000^{\circ} \mathrm{C}$. The components in the switchboard can only withstand this temperature within 250 milliseconds before sustaining severe damages [10]. 


\section{Damage vs. Time and Current}

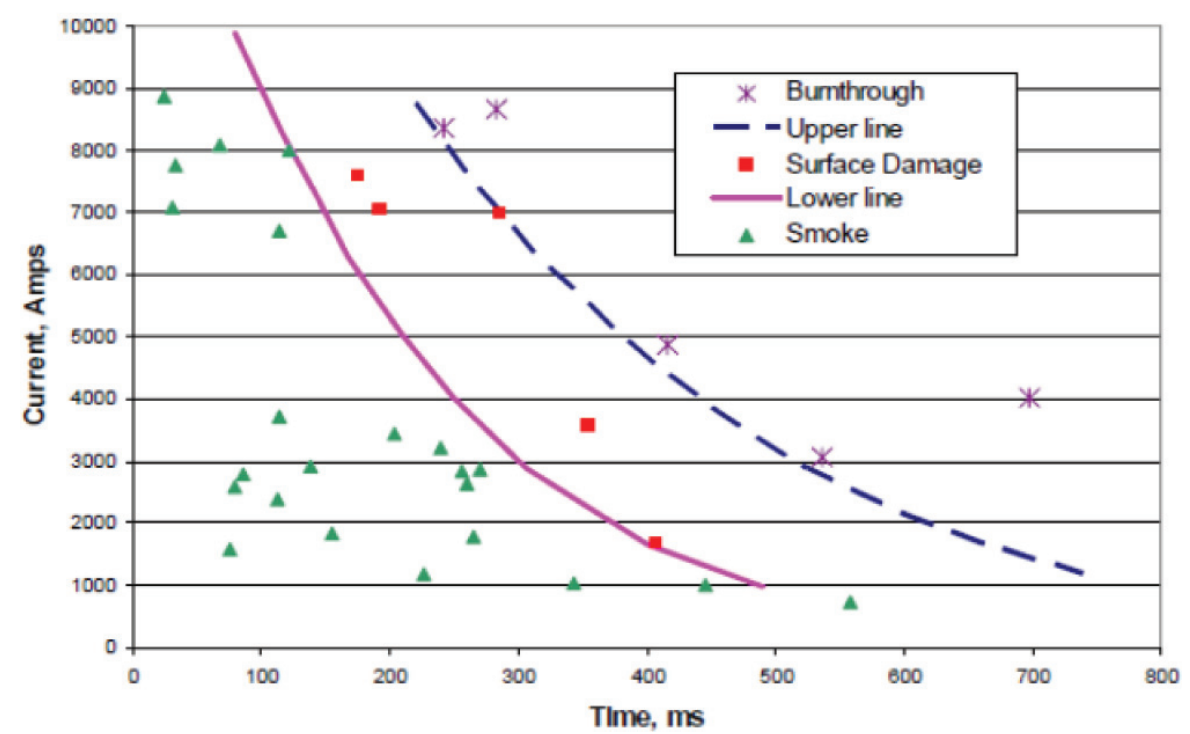

Figure 1 Damage to the side of a switchboard versus arc current and time.

The design of light detector is based on the principle that during an arc flash, large amount of light will be detected by the detector to indicate a flash. An arc flash can draw a fraction of bolted-fault current, especially in the early stages, circuit breakers alone cannot be relied upon to differentiate between the arcing current and a typical short-circuit current. By incorporating a light detector in the arc fault detection circuit, it reduces the total clearing time and the amount of energy released through an arcing fault greatly [11].

\section{System Description of Arc Fault Detection Circuit}

Figure 2 shows the block diagram for the proposed design of the arc fault detection circuit. It consists of a pressure detector, a temperature detector, a light detector, voltage comparator, an AND gate, a relay and a LED. The pressure, temperature and light detectors will detect the arc fault by amount of pressure heat and the light produced and convert into corresponding voltage and the output of voltage comparator from these sensors will send to the AND gate. A 'HIGH' output from the AND gate will trigger the relay to send a trip signal that will turn on the LED. 


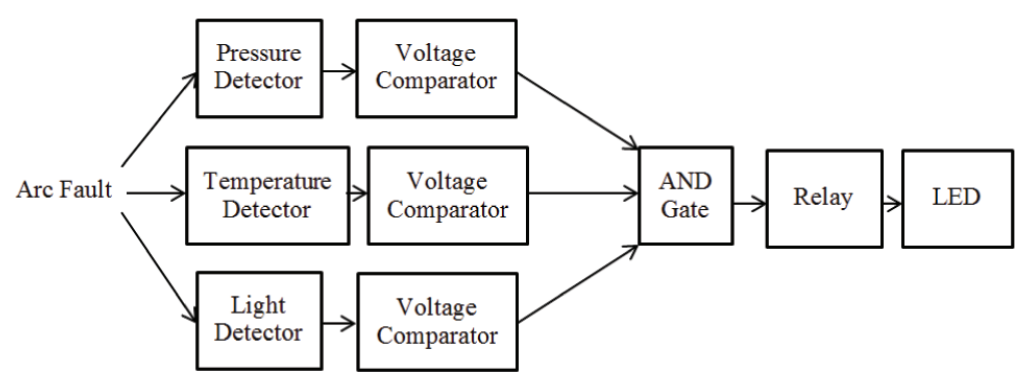

Figure 2 Block diagram of an arc fault detection circuit.

The 1140 pressure sensor is used to detect the presence of an arcing fault by sensing the pressure changes in the switchboard. 1140 has a breakdown voltage directly proportional to the temperature, which is $+12 \mathrm{mV} / \mathrm{kPa} .1140$ is chosen because the 1140 Absolute Pressure Sensor is an air pressure sensor that measures the absolute pressure of its environment. It can measure pressures from $20 \mathrm{kPa}$ to $400 \mathrm{kPa}$. In addition, it has a high precision and narrow measurement range compared to other types of pressure sensors. It has typically less than $\pm 1.5 \%$ error. The pressure range for 1140 is $20 \mathrm{kPa}$ to $400 \mathrm{kPa}$. In other words, the output voltage of this pressure sensor will range from $240 \mathrm{mV}$ to $4.8 \mathrm{~V}$.

The calculations for the output voltage are shown below:

$$
\begin{aligned}
& \text { Output voltage for } 20 \mathrm{kPa}=20 \times 12 \times 10^{-3}=0.24 \mathrm{~V} \\
& \text { Output voltage for } 400 \mathrm{kPa}=400 \times 12 \times 10^{-3}=4.8 \mathrm{~V}
\end{aligned}
$$

Every $1 \mathrm{kPa}$ increases in the pressure of the surrounding. The output voltage will increase by $12 \mathrm{mV}$. Since the atmospheric pressure is $100 \mathrm{kPa}$, it is assumed that under normal condition, the pressure inside a switchboard is about $150 \mathrm{kPa}$ which is equivalent to $1.8 \mathrm{~V}$. From [1], a true arc fault will rapidly increase in pressure up to $3 \mathrm{~atm}$ or equivalently to $303.975 \mathrm{kPa}$. Therefore, in this proposed design, the pressure of $250 \mathrm{kPa}$ is set as the reference value. By calculation, the voltage value corresponding to $250 \mathrm{kPa}$ is $3.0 \mathrm{~V}$. This voltage is set as the reference voltage. The input voltage (i.e. the pressure inside the switchboard) will be compared with the reference voltage (i.e. the reference pressure value of $250 \mathrm{kPa}$ ). A "HIGH" output from the voltage comparator will send to the AND gate if the input voltage is higher than the $3.0 \mathrm{~V}$.

The LM 335 temperature sensor is used to detect the presence of an arcing fault by sensing the temperature changes in the switchboard. LM 335 has a breakdown voltage directly proportional to the temperature, which 
is $+10 \mathrm{mV} /{ }^{\circ} \mathrm{K}$. LM 335 is chosen because it is precise, easily calibrated and integrated circuit temperature sensor. In addition, it has a linear output and it is cheaper compared to other types of temperature sensors. When it is calibrated at $25^{\circ} \mathrm{C}$, it has typically less than $1^{\circ} \mathrm{C}$ error over a $100^{\circ} \mathrm{C}$. The temperature range for LM 335 is $-40^{\circ} \mathrm{C}$ to $100^{\circ} \mathrm{C}$. In other words, the output voltage of this temperature sensor will range from $2.33 \mathrm{~V}$ to $3.73 \mathrm{~V}$.

The calculations for the output voltage are shown below [6]:

$$
\begin{array}{r}
\text { Output voltage for }-40^{\circ} \mathrm{C}=(-40+273) \times 10 \times 10^{-3} \\
=2.33 \mathrm{~V}
\end{array}
$$

Output voltage for $100^{\circ} \mathrm{C}=(100+273) \times 10 \times 10^{-3}=3.73 \mathrm{~V}$

For every $1^{\circ} \mathrm{K}$ increase, the output voltage will increase by $10 \mathrm{mV}$. Under normal condition, the temperature inside a switchboard is about $40^{\circ} \mathrm{C}$. It is assumed that the temperature inside a switchboard will rise to $92^{\circ} \mathrm{C}$ and by calculation; the corresponding voltage is $3.65 \mathrm{~V}$. This voltage is set as the reference voltage. The input voltage will be compared with the reference voltage. The relay will be activated, once the input voltage is more than the reference voltage.

The ISL29101 light to analog sensor is used to detect the presence of an arcing fault by sensing the illumination (lux) level in the interior of the switchboard. It is able to detect up to an illumination level of 10000 lux. The corresponding output voltage for 0 lux to $100 \mathrm{lux}$ is $1 \mathrm{mV}$ to $1.65 \mathrm{~V}$. Therefore, for every llux increase, the corresponding voltage is $16.5 \mathrm{mV}$.

Since the illumination level for the switchroom from IES standard is 300 lux [12], it is assumed that under normal condition, the illumination level in the interior of the switchboard is about 50 lux which is equivalent to $0.825 \mathrm{~V}$. In this proposed design, the illumination level of 90 lux is set as the reference value. And the voltage value corresponding to 90 lux is $1.5 \mathrm{~V}$. This voltage is set as the reference voltage. The input voltage (i.e. the illumination level inside the switchboard) will be compared with the reference voltage (i.e. the reference illumination level of 90 lux). A "HIGH" output from the voltage comparator will send to the AND gate if the input voltage is higher than the $1.5 \mathrm{~V}$.

\section{Modelling of Arc Fault Detection Circuit}

A buffer amplifier provides electrical impedance transformation from one circuit to another circuit. It is used to transfer a voltage from the light sensor, pressure sensor and temperature sensor to the voltage comparator. A unity 
gain buffer is used in the circuit design. The output of the op-amp (buffer) is connected to its inverting input, which is the negative feedback. Therefore, the output voltage is simply equal to the input voltage of the buffer. The outputs from the pressure sensor, temperature sensor and light sensor are connected to the non-inverting input of the buffer (op-amp), which is the positive feedback, and the output from the buffer is identical to the these sensors.

The pressure sensor, temperature sensor and light sensor will generate a voltage based signal with respect to the amount of pressure, temperature and light detected from the interior of the switchboard. The signal is then sent to a voltage comparator through a buffer. The voltage comparator is used to compare the signal with a reference voltage. The output of the comparator from all these sensors will produce a positive ('HIGH' output) value which will then become the inputs for the 7410 AND gate. Then the output of the AND gate will activate the relay and send a trip signal to the circuit breaker (i.e.to turn on the LED) if all the light sensor, pressure sensor and temperature sensor detect an illumination level, pressure and temperature that exceed the reference voltage of the voltage comparator. Else, the output voltage of the voltage comparator will indicate a negative value ('LOW' output) which will not trigger the trip signal.

Before detect the changes of light, pressure and temperature in the interior of switchboard and operate the LED when the predetermined limit is exceeded, these detectors are modeled to lower values of pressure and temperature with respect to the practical illumination level, pressure and temperature value.

The schematic diagram of the pressure detector, temperature detector and light detector in PSpice program are shown in Figures 3-5 respectively.

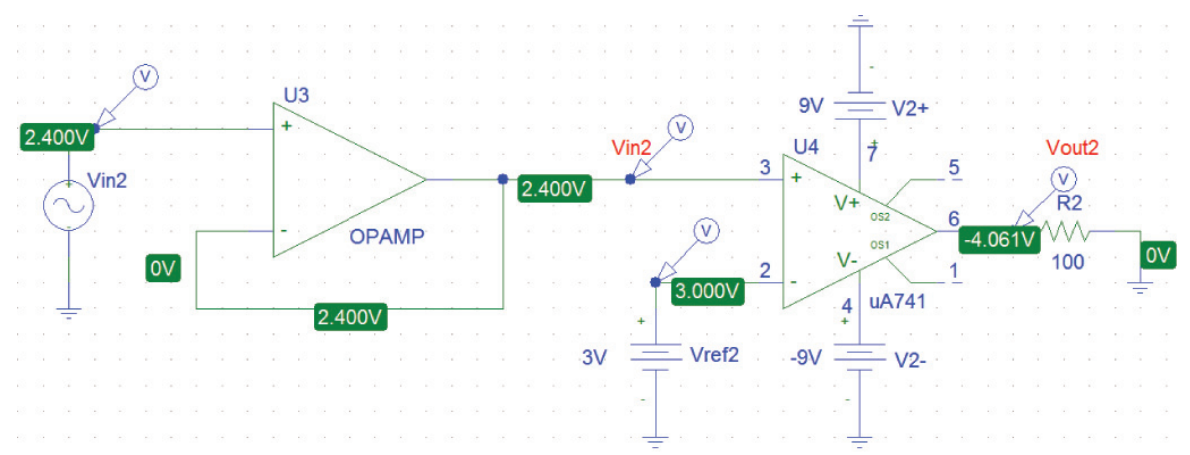

Figure 3 PSpice schematic diagram of the arc fault pressure detector. 


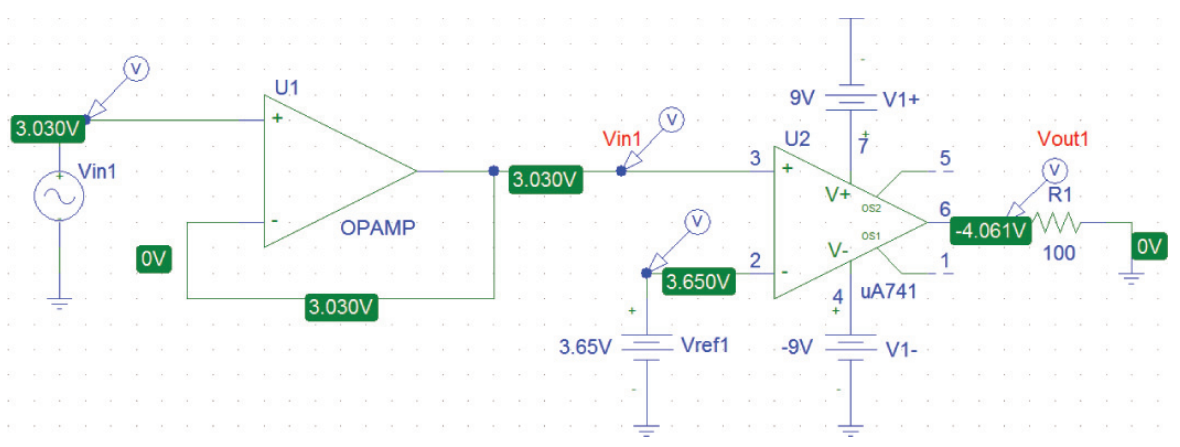

Figure 4 PSpice schematic diagram of the arc fault temperature detector.

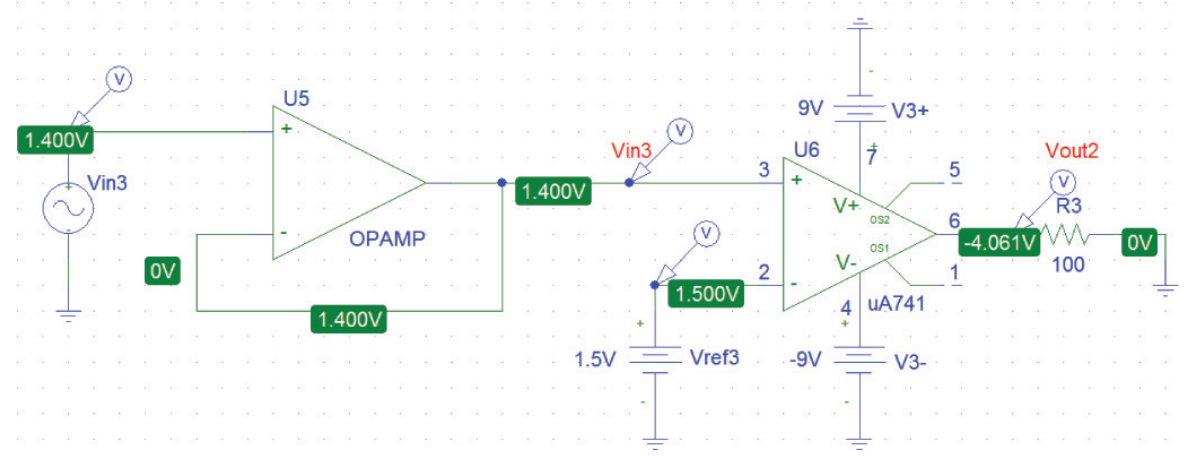

Figure 5 PSpice schematic diagram of the arc fault light detector.

Figure 6 shows the schematic diagram of the overall arc fault detection circuit using PSpice program. The input for this circuit is an AC supply. An AC supply is used to represent the output signal from the light, pressure and temperature sensor. The output voltage range of the sensor is used as the input voltage range for the circuit. The $\mathrm{AC}$ input voltage for temperature sensor, Vin 1, is ranged from $2.33 \mathrm{~V}$ (corresponding to $-40^{\circ} \mathrm{C}$ ) to $3.73 \mathrm{~V}$ (corresponding to $100^{\circ} \mathrm{C}$ ) as obtained from Equation (3) and Equation (4). The $\mathrm{AC}$ input voltage for pressure sensor, Vin2, is ranged from $0.2 \mathrm{mV}$ (corresponding to $20 \mathrm{kPa}$ ) to $4.8 \mathrm{~V}$ (corresponding to $400 \mathrm{kPa}$ ) as obtained from Equation (1) and Equation (2). The AC input voltage for temperature sensor, Vin3, is ranged from $0 \mathrm{~V}$ (corresponding to $0 \mathrm{lux}$ ) to $1.65 \mathrm{~V}$ (corresponding to $100 \mathrm{lux}$ ).

$\mathrm{U} 1, \mathrm{U} 3$ and U5 are an op-amp, which represents a buffer in this circuit. The AC supply is connected to the positive feedback of U1, U3 and U5 and the negative feedback of $\mathrm{U} 1$ and $\mathrm{U} 3$ is connected to the output of $\mathrm{U} 1, \mathrm{U} 3$ and 


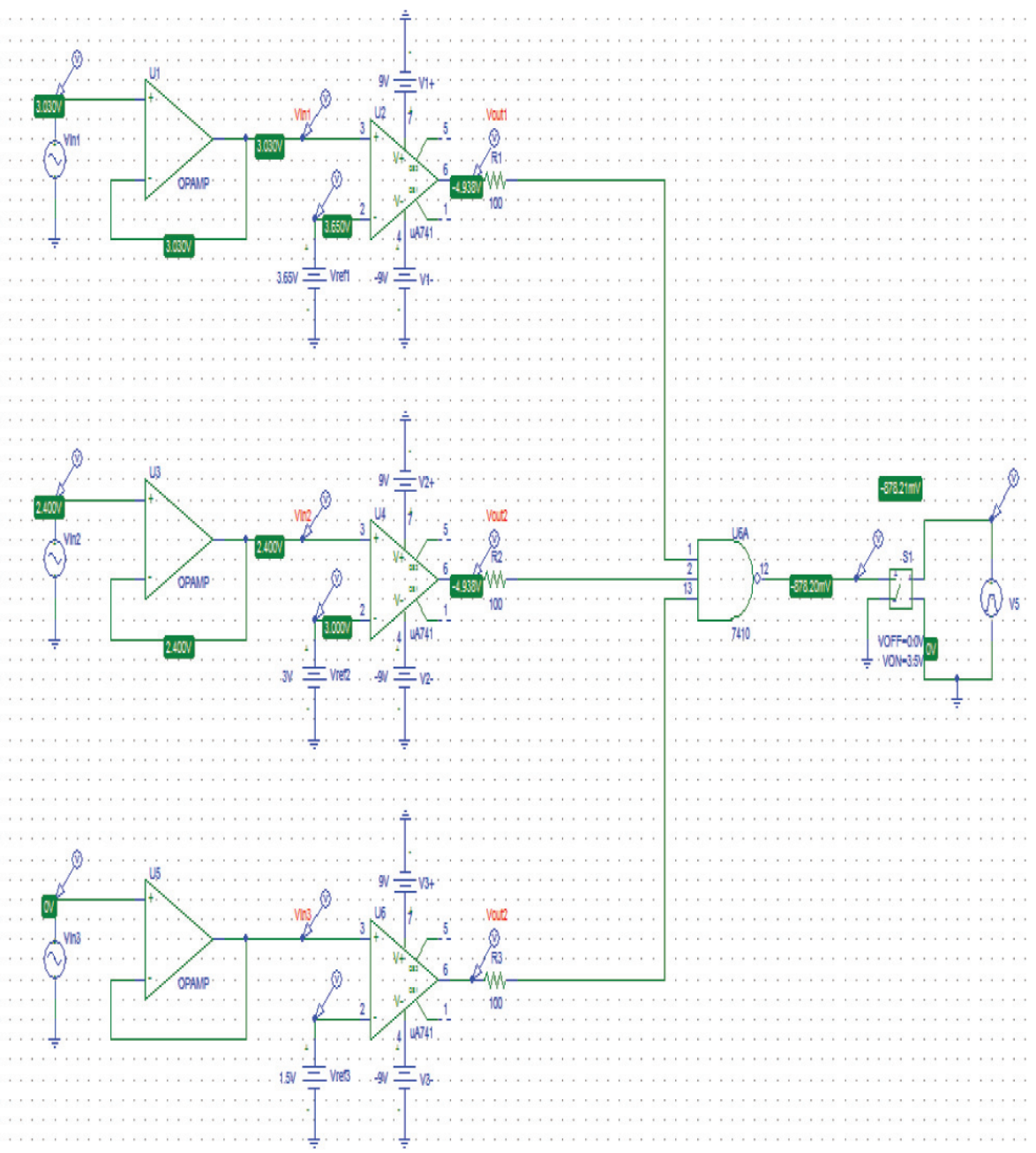

Figure 6 PSpice schematic diagram of the overall arc fault detection circuit.

U5 to produce a unity gain buffer. The output voltage of U1, U3 and U5 are same as the input voltage since it is a unity gain buffer.

Then, the output voltage of U1, Vin1, is connected to the positive feedback (pin 3) of U2, which is a voltage comparator. Also, the output voltage of U3, Vin2, is connected to the positive feedback (pin 3) of U4, which is a voltage comparator. The output voltage of U5, Vin3, is connected to the positive feedback (pin 3) of U6, which is a voltage comparator. 
The output voltage of the buffer is used as the input voltage of the comparator. Theoretically, the input voltage of U2, U4 and U6 are identical to the output voltage of U1, U3 and U5 and are also identical to the output voltage of the light, pressure and temperature sensor, which is represented by an AC source in this circuit. uA741 op-amp is used as the voltage comparator. A DC input voltage, Vref1, of $3.65 \mathrm{~V}$ is placed at the negative feedback (pin 2) of $\mathrm{U} 2$ to produce a constant value of reference voltage. The voltage value of $3.65 \mathrm{~V}$ is equivalent to the temperature value of $92^{\circ} \mathrm{C}$. Also, a DC input voltage, Vref2, of $3 \mathrm{~V}$ is placed at the negative feedback (pin 2) of U4 to produce a constant value of reference voltage. The voltage value of $3 \mathrm{~V}$ is equivalent to the pressure value of $250 \mathrm{kPa}$. For the light detector circuit, a DC input voltage, Vref3, of $1.5 \mathrm{~V}$ is placed at the negative feedback (pin 2) of U6 to produce a constant value of reference voltage. The voltage value of $1.6 \mathrm{~V}$ is equivalent to the illumination value of 90 lux.

A +9 V DC supply is connected to pin 7 and a -9 V DC supply is connected to pin 4 of U2, U4 and U6 to supply voltage for this component. Output voltage from U2, U4 and U6 (pin 6), Vout1, Vout2 and Vout3, is used to indicate the comparison result of the input voltage and the reference voltage.

The 7410 AND Gate with three inputs are connected to the outputs voltage of U2, U4 and U6 then AND Gate output is connected to the Voltage Controlled Switch which act as a relay that will activate the Voltage Pulse and therefore turn on the LED if output voltage of the AND gate is positive.

\section{Simulation Results}

The PSpice simulation results from the schematic diagram of the pressure, temperature and light detectors are shown in Figures 7-9 respectively.

The straight line in red color in Figure 9 represents the reference voltage of $1.5 \mathrm{~V}$ for the light sensor. The sine wave in purple color is the input signal that used to trigger the light sensor. The square wave is the output from voltage comparator which trigger as logic ' 1 ' ('HIGH') at $4.0 \mathrm{~V}$ if the input voltage of sine wave is higher than the reference voltage and becomes logic ' 0 ' ('LOW') at $-4.0 \mathrm{~V}$ if the input voltage of sine wave is lower than the reference voltage.

Figure 10 shows the PSpice simulation result from the schematic diagram of the arc fault pressure and temperature detectors circuit. As can be seen in Figure 10, the input voltage of temperature sensor of $3.03 \mathrm{~V}$ is represented by the sine waveform (in pink color) and input voltage of pressure sensor of $2.4 \mathrm{~V}$ is represented by another sine waveform (in light blue color). The reference voltages are the two straight lines at $3.65 \mathrm{~V}$ for temperature sensor 


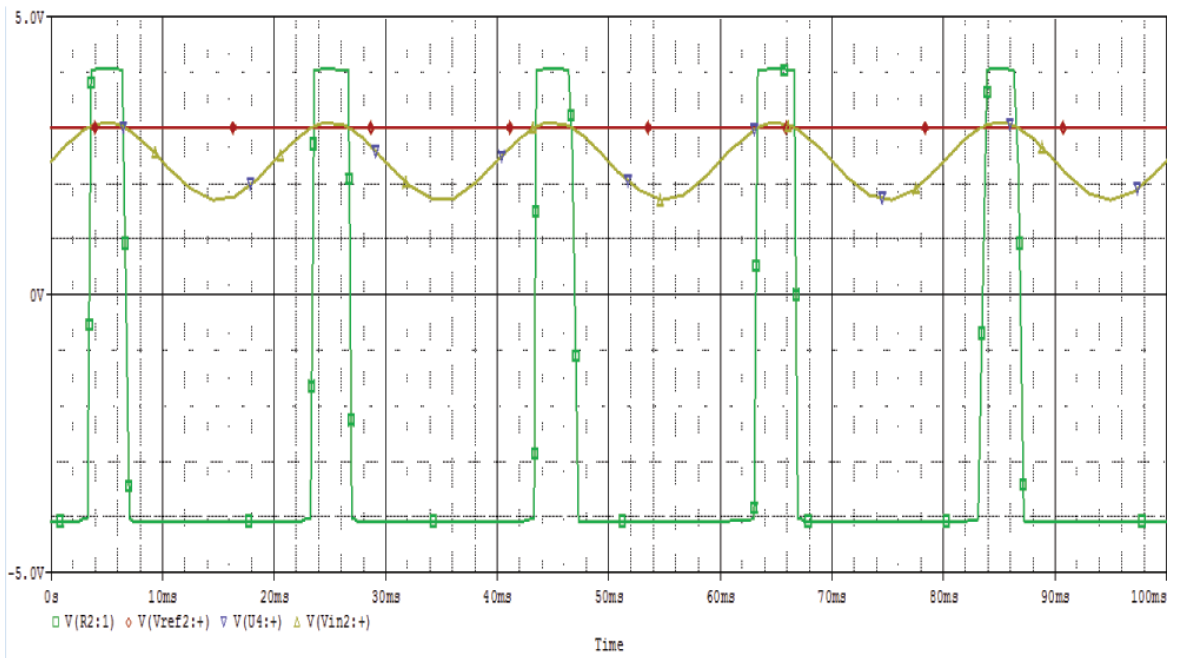

Figure 7 Simulation result of the arc fault pressure detector.

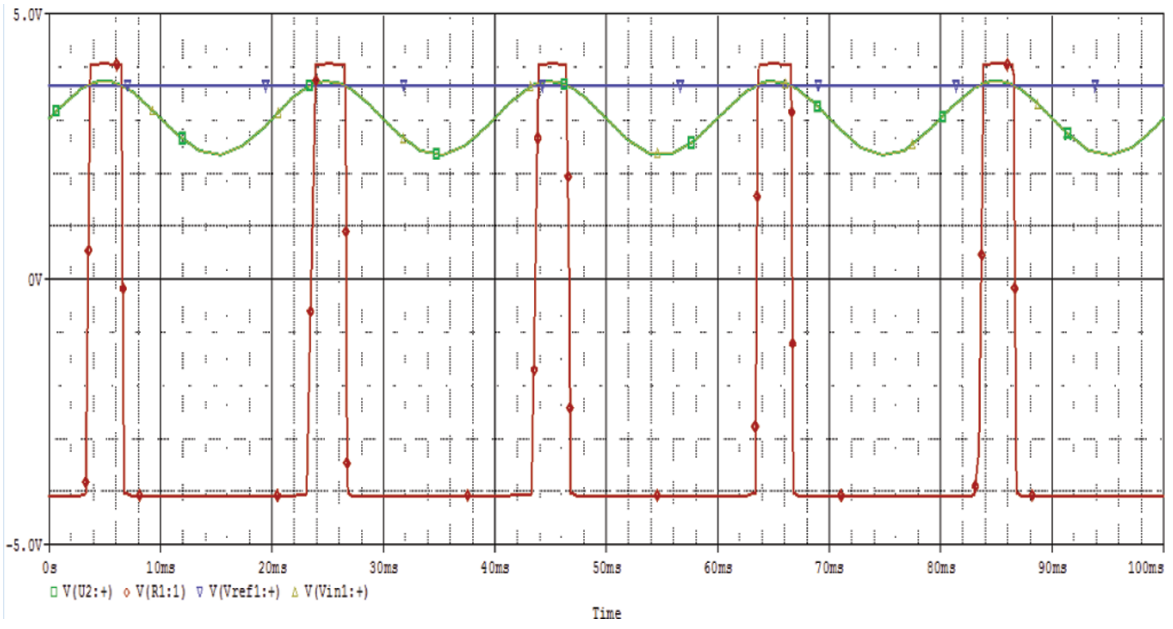

Figure 8 Simulation result of the arc fault temperature detector.

and 3.0 V for pressure sensor. The two square waves are the output from voltage comparator which trigger as logic ' 1 ' ('HIGH) at $8.5 \mathrm{~V}$ if the input voltage of sine wave is higher than the reference voltage and becomes logic ' 0 ' ('LOW') at $-5.0 \mathrm{~V}$ if the input voltage of sine wave is lower than the reference voltage. 


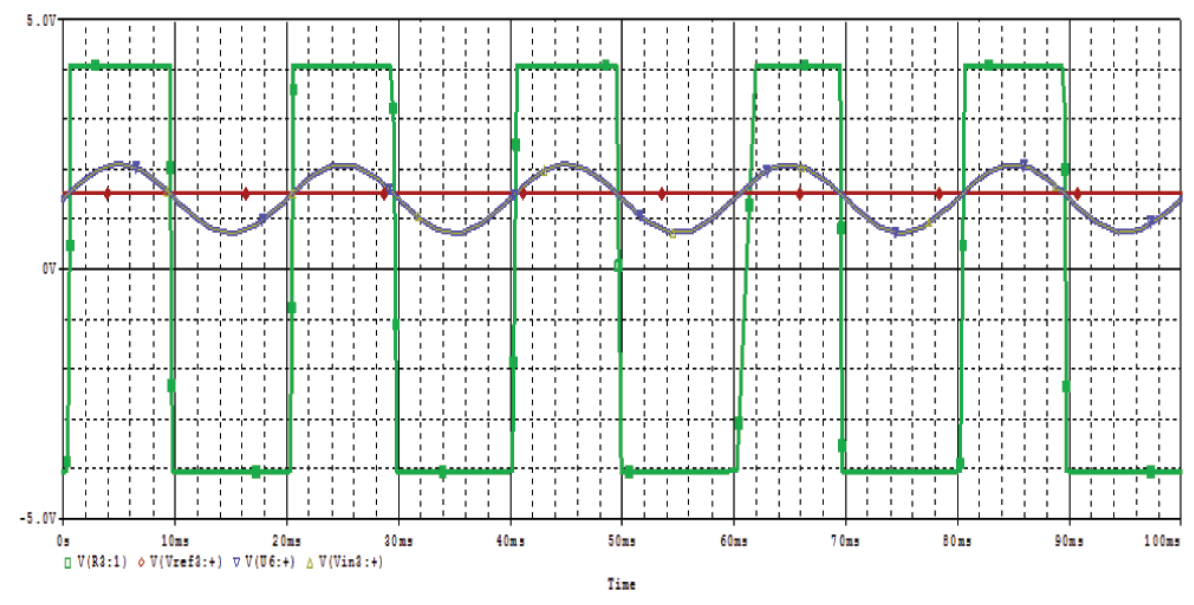

Figure 9 Simulation result of the arc fault light detector.

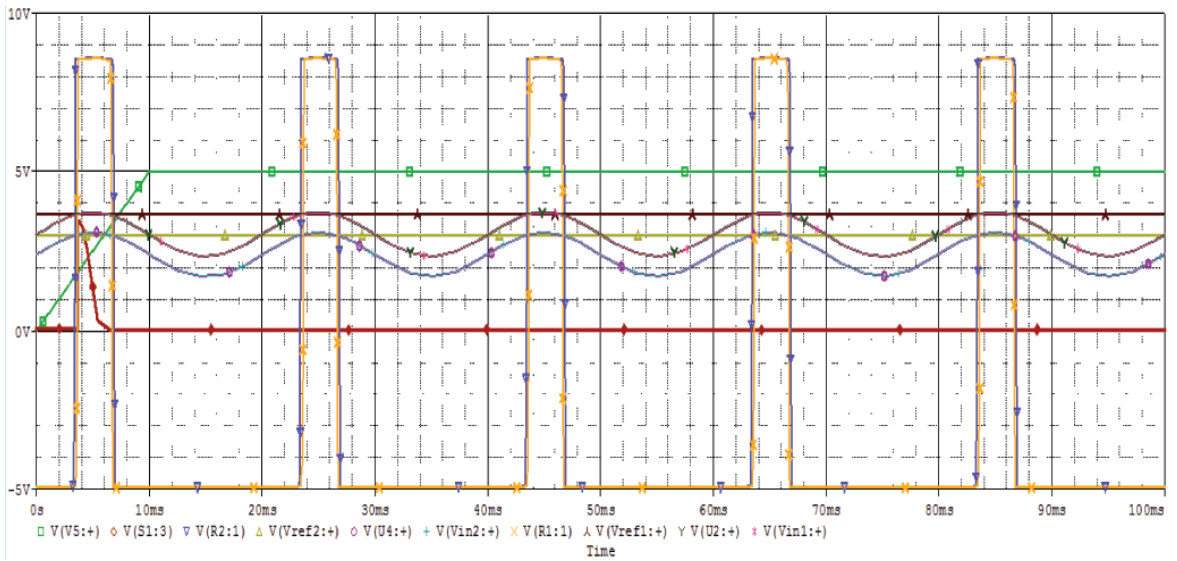

Figure 10 Simulation result of arc fault pressure and temperature detectors.

The red line at $3.5 \mathrm{~V}$ between the times of $0 \mathrm{~ms}$ to $10 \mathrm{~ms}$ is the output voltage of AND gate which combine the output voltage of pressure and temperature detector. After $10 \mathrm{~ms}$ the red line becomes $0 \mathrm{~V}$ as it trip the signal at the voltage controlled switch. The voltage controlled switch is act like a relay and will close when it receive a 'HIGH' output from the AND gate. Equivalently, it will trigger the voltage pulse at $5.0 \mathrm{~V}$ (represent in green color) which then turn on the LED. 
Unfortunately, there is some limitation on the PSpice software due to the analog node limit is exceeded when we run the overall circuit with these three detectors. However, from the simulation results obtained from the individual detector, it is expected that the controlled voltage switch will receive a 'HIGH' output from the AND gate when the output from these three detectors output is sent to the 7410 AND gate. Then the voltage controlled switch will act like a relay and close to complete the circuit and allows the voltage pulse triggers at 5.0 V. Then, the LED will turn on.

\section{Conclusion}

Arcing fault in low voltage switchboard is a serious issue as the effects of the arcing faults are devastating. In this paper, the proposed arc fault detection circuit will activate the relay and send a trip signal to the circuit breaker if and only if all three signals, i.e. pressure, thermal and light are detected. With these three arc fault signals prior the occurrence of arc, the proposed design is able to ensure that no fault tripping signal is sent to the circuit breaker and therefore no unnecessary power shut down.

An early detection of arc fault in low voltage switchboard enable the isolation of the power supply to the consumer side just before the occurrence of arc fault and thereby reduce the danger to personal injury and building. In addition, it improves the system reliability without power interruption which is particular essential to hospitals and certain industries with sensitive loads. The circuit help to eliminate the possibility of an arc occurring and hence prevents against the effects of arc occurrence. The proposed circuit can be modeled to meet specifications of industry with different supply requirements. It is easy to design and highly reliable.

\section{References}

[1] Gammon, T., and Mattews, J. (1999) "The historical evolution of arcing-fault models for low voltage systems," in Proceedings of the IEEE Industrial \& Commercial Power Systems Technical Conference, Detroit, MI.

[2] Kuan, L. C. (2013). Arc fault pressure detector in low voltage switchboard. Int. J. Sci. Res. Pub. 3.

[3] Wilson, R. A. (ABB Inc.), Harju, R. (ABB Oy, Finland), Keisala, J. (ABB Oy, Finland), and Ganesan, S. (ABB Ltd., India), "Tripping with the Speed of Light: Arc Flash Protection". 
14 K. L. Choo and A. A. Sa'adon

[4] Knapek, W., OMICRON Electronics Corp. Zeller, M., and Schweitzer Engineering Laboratories, Inc., (2011). Verify Performance and Safety of Arc-Flash Detection Systems. Pullman, WA: Schweitzer Engineering Laboratories, Inc.

[5] Land, H. B. (2008). The behavior of arcing faults in low voltage switchboards. IEEE Trans. Ind. Appl. 44, 437-444.

[6] Malmedal, K., and Sen, P. K. (2000). "Arcing fault current and the criteria for setting ground fault relays in solidly-grounded low voltage systems," Proceedings of the Industrial and Commercial Power Systems Technical Conference, Detroit, MI.

[7] Gammon, T., and Matthews, J. (2000). "Arcing fault models for low voltage power systems," in Proceedings of the Industrial and Commercial Power Systems Technical Conference, Detroit, MI.

[8] Kuan, L. C. (2015). "Arc fault temperature detector in low voltage switchboard," in Proceedings of the International Conference of Information, System and Convergence Applications, Kuala Lumpur.

[9] Baliga, B. R., and Pfender, E. (1975). Fire Safety Related Testing of Electric Cable Insulation Materials. Minneapolis, MN: University of Minnesota.

[10] Land, H. B., Eddins, C. L., and Klimek, J. M. (2004). Evolution of Arc Fault Protection Technology. Washington, DC: John Hopkins APL Technical Digest.

[11] Seedorff, J. (2015). Arc Flash Protection: Key Considerations for Selecting An Arc Flash Relay. Chicago, IL: Littelfuse, Inc.

[12] "Room Illumination level: General Building Areas", IES Standards, MS 1525 . 


\section{Biographies}

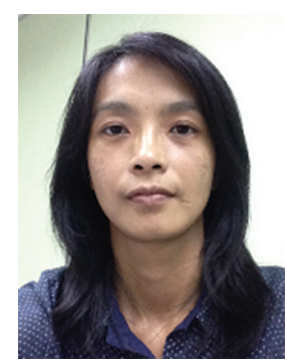

K. L. Choo received the Bachelor of Engineering from University Tenaga Nasional, Malaysia and M.Sc. degree from Imperial College, London, in 2001 and 2002 respectively. She is a Professional Engineer of Board of Engineers Malaysia (BEM) and Corporate Member of The Institution of Engineers Malaysia (IEM). Currently, she works as a lecturer in Infrastructure University Kuala Lumpur, Malaysia. Her research interests include control systems, power quality and electrical power system.

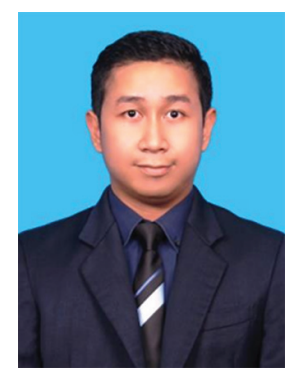

A. A. Sa'adoni received the Bachelor of Electronics Engineering (Hons) from Infrastructure University Kuala Lumpur, Malaysia in 2016. Currently, he works as a project engineer in SRS Power Engineering Sdn. Bhd. (an established low voltage switchboard company). He has major experience and knowledge in electrical power system (infrastructure), telecommunication (fiber optics) and power electronics (arc fault). 
